\title{
High-Resolution Correlative Microscopy: Bridging the Gap between Single Molecule Localization Microscopy and Atomic Force Microscopy
}

\author{
Pascal D. Odermatt, ${ }^{\dagger}$ Arun Shivanandan, ${ }^{\ddagger}$ Hendrik Deschout, ${ }^{\ddagger}$ Radek Jankele, ${ }^{\ddagger}$ Adrian P. Nievergelt, ${ }^{\dagger}$
} Lely Feletti, ${ }^{\ddagger}$ Michael W. Davidson, ${ }^{\S}$ Aleksandra Radenovic, ${ }^{*}, \neq$ and Georg E. Fantner ${ }^{*}$,

${ }^{\dagger}$ Laboratory for Bio- and Nano-Instrumentation, Institute of Bioengineering and Institute of Microengineering and ${ }^{\ddagger}$ Laboratory of Nanoscale Biology, Institute of Bioengineering, School of Engineering, EPFL, 1015 Lausanne, Switzerland

${ }^{\S}$ National High Magnetic Field Laboratory and Department of Biological Science, Florida State University, Tallahassee, Florida 32306 United States

\section{Supporting Information}

ABSTRACT: Nanoscale characterization of living samples has become essential for modern biology. Atomic force microscopy (AFM) creates topological images of fragile biological structures from biomolecules to living cells in aqueous environments. However, correlating nanoscale structure to biological function of specific proteins can be challenging. To this end we have built and characterized a correlated single molecule localization microscope (SMLM)/AFM that allows localizing specific, labeled proteins within high-resolution AFM images in a biologically relevant context. Using direct stochastic optical reconstruction microscopy (dSTORM)/AFM, we directly correlate and quantify the density of localizations with the $3 \mathrm{D}$ topography using both imaging modalities along (F-)actin cytoskeletal filaments. In addition, using photo activated light microscopy (PALM)/AFM, we provide correlative images of bacterial cells in aqueous conditions. Moreover, we report the first correlated AFM/PALM imaging of live mammalian cells. The complementary information provided by the two techniques opens a new dimension for structural and functional nanoscale biology.

KEYWORDS: Atomic force microscopy (AFM), single molecule localization microscopy (SMLM), direct stochastic optical reconstruction microscopy (dSTORM), correlative imaging, photoactivated localization microscopy (PALM), live cell imaging

$\mathrm{O}$ bserving nanoscale structural changes associated with many cellular processes is essential for understanding the complex mechanisms underlying biomolecular function. Performing the experiments in aqueous environments is essential for maintaining cellular integrity. At the nanoscale, the two imaging modalities atomic force microscopy (AFM) and single molecule localization microscopy (SMLM) fulfill the requirement of concomitant assessment of structures and dynamics under close to physiological conditions. AFM has long since provided nanoscale structural information on living cells. ${ }^{1-3}$ Recent advances in high-speed AFM have even been successfully used to directly visualize the dynamics of molecular machinery such as protein motors. ${ }^{4,5}$ In addition, newly emerged SMLM techniques extended all the advantages of fluorescence microscopy beyond its diffraction limited spatial resolution of about $200 \mathrm{~nm}^{6-8}$ and provided new important insights into structural organization, ${ }^{9,10}$ dynamics, ${ }^{11}$ and quantification $^{12-14}$ of biomolecules. Both techniques by themselves have proven to be very powerful for research in nanoscale structural biology. However, in SMLM, biomolecules themselves are invisible, and one observes/localizes only the fluorophores. In addition, translating the SMLM localization precision, typically between 10 and $25 \mathrm{~nm}$, into spatial resolution is still very challenging due to a number of complicating factors. ${ }^{15}$ Therefore, early on, the validation of SMLM has been carried out with correlative imaging using transmission electron microscopy (TEM). ${ }^{6}$ Yet, in the case when SMLM is correlated either with TEM $^{6,16}$ or scanning electron microscopy (SEM), ${ }^{17,18}$ sample preparation conditions are far from physiological, prohibiting the observation of live processes. Structural artifacts might as well be introduced unless one performs experiments on vitrified samples as Chang et al. $^{16}$

AFM on the other hand has proven to obtain submolecular resolution on membrane proteins in solution, ${ }^{19}$ but it fails at providing biomolecular specificity. In combination, AFM and SMLM hold promise for doing correlated nanoscale biomolecular and structural characterization on living samples in physiological conditions.

Nevertheless, the first attempts to correlate AFM with SMLM $^{18}$ have not fully exploited this opportunity. For example, Monserrate et al. ${ }^{18}$ performed AFM imaging of

Received: February 10, 2015

Revised: June 22, 2015 
DNA in air, dried on a mica substrate. While this approach allows a nice comparison of the two techniques, it does not allow for the two techniques to complement each other to learn new things about structure-function relationships in living systems. In addition, the presence of the thin mica sheet on the glass coverslip deteriorated the point spread function (PSF) and compromised the performance of SMLM. Although Chacko et al. $^{20}$ employ both modalities in physiological conditions, their samples were chemically fixed.

In order to exploit the full potential of high-resolution correlative AFM/SMLM imaging, one has to maintain optimal performance in both imaging modalities. This requires careful design of experimental procedures so that the two methods do not deteriorate each other's performance. In particular, during AFM imaging, maintaining low imaging forces is essential to ensure minimal damage to the soft biological samples in fluid. At the same time, one has to maintain a low level of noise that should not be compromised by the integration with the SMLM optical microscope. To this end, we have built a mechanical support structure to hold the AFM in place without it mechanically contacting the microscope body (Figure 1). Simultaneously, in SMLM it is important that the fluorescent labeling strategy minimally affects the 3D structure of the sample. Due to this requirement, from the plethora of labeling/ imaging strategies we have chosen direct stochastic optical reconstruction microscopy (dSTORM) and photo activated light microscopy (PALM). In dSTROM, the light-induced reversible photoswitching of organic fluorophores is obtained in the presence of millimolar concentrations of reducing thiol compounds such as dithiothreitol (DTT), glutathione (GSH), or mercaptoethylamine (MEA), but without requiring the presence of an activator fluorophore in aqueous buffer. ${ }^{21}$ In particular, using fusion proteins or intercalating small molecules such as fluorescently labeled phalloidin, allowed us to completely avoid antibodies that would have otherwise significantly altered the sample's 3D structure. Also, after ensuring that the laser used by the AFM to detect the cantilever motion does not overlap with the fluorophore excitation spectra, only a subset of fluorophores is available for correlated microscopy.

The purpose of correlated AFM/dSTORM microscopy can be two-fold: first to compare and validate the resolution of one technique with the other, and second to provide complementary information about two different aspects of the same biological sample. To establish accurate correlation and measurement protocols, we chose F-actin as a first test sample, since high-resolution images of F-actin have been reported in both imaging modalities. Monomers in actin filaments are very weakly bound since they have to be dynamically assembled and disassembled in the cell. Even after stabilization by phalloidin they are very delicate. AFM imaging of actin filaments in physiological buffer therefore requires excellent force control to image at high resolution without destroying the fibers. ${ }^{22}$ This makes them an excellent test sample to ensure high-quality AFM performance. Actin is also one of the best studied structures with SMLM both in vitro ${ }^{23}$ and in vivo. ${ }^{24}$

For direct comparison of the two techniques we imaged polymerized actin filaments on a glass coverslip. The sample preparation procedure was designed to meet the requirements of both techniques. Fluorescently labeled phalloidin was used to prepare the sample for SMLM as well as for AFM, although not strictly required in the latter case. The glass coverslip was functionalized with APTES ((3-aminopropyl)triethoxysilane)
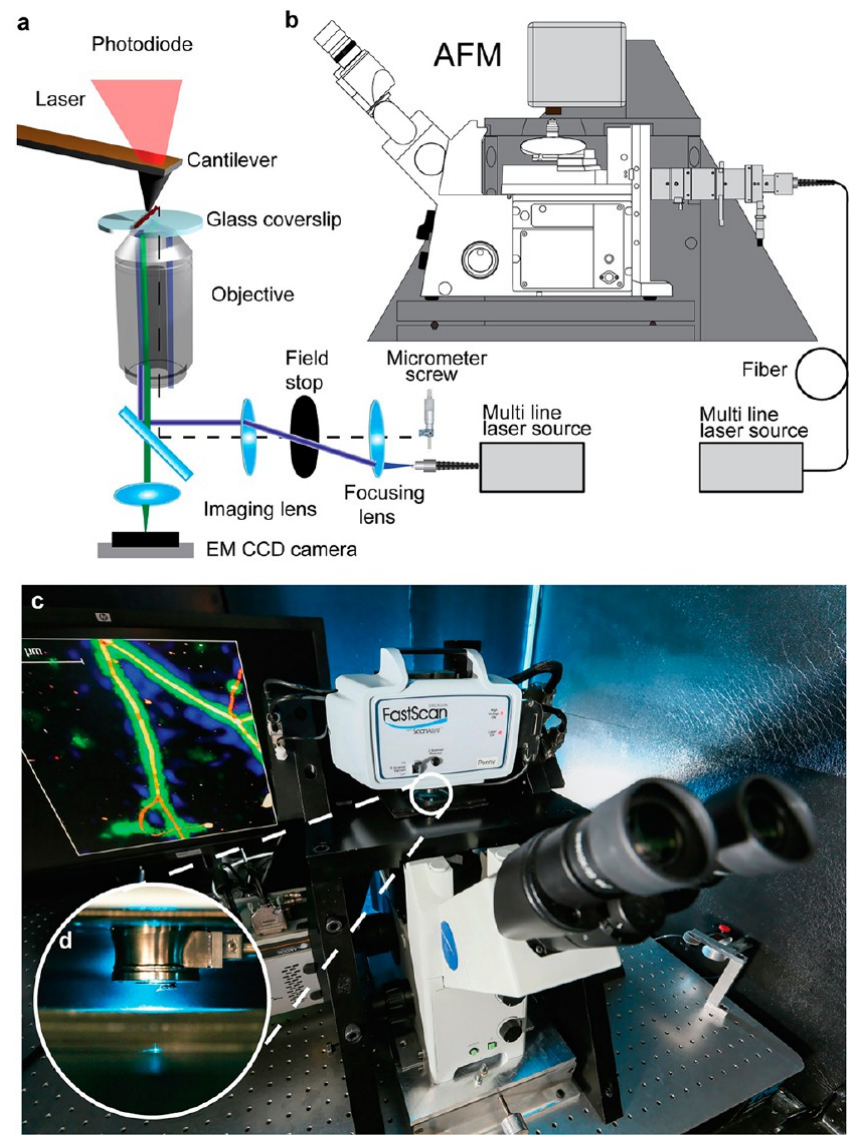

Figure 1. Correlative AFM-SMLM: instrument setup. (a) Schematic of the aligned optical path with the AFM cantilever. By laterally translating the incoming laser beam using a micrometer screw, the TIRF illumination condition is enabled. The AFM cantilever is centered in the field of view by adjusting the position of the inverted optical microscope mounted on an $x / y$-translation stage (as shown in $\mathrm{b}$ and c). (b) Mechanical integration of an inverted optical microscope and the AFM. The inverted optical microscope is mounted on an $x / y$ translation stage. Around it a mechanical support structure is built to hold the AFM in place without mechanically contacting the microscope body. The whole instrument is placed on a vibration isolation platform inside an acoustic isolation box. (c) Photograph of the instrument and (d) zoom in to the AFM cantilever aligned to the optical axis.

to adhere the actin filaments as required for AFM and SMLM imaging. Both imaging modes were performed in buffer; however, each technique requires its own. While the SMLM experiment requires a buffer optimized for blinking of the corresponding fluorescent dye, the AFM requires a buffer which ensures structural integrity and stability.

The best image quality by AFM was obtained using soft cantilevers (BL-AC40TS-C2 cantilevers (Olympus)) with a nominal spring constant of $0.09 \mathrm{~N} / \mathrm{m}$ operated in peak force tapping keeping the force set point below $100 \mathrm{pN}$ to not destroy the filament. Figure 2a shows a high-resolution AFM image of the F-actin, revealing the $\sim 36 \mathrm{~nm}$ periodicity of the single left handed generic helix. ${ }^{25}$ The spacing of the individual $\mathrm{G}$-actin monomers can be seen in the cross section (Figure 2c), which is equivalent to the highest resolution AFM images of Factin published thus far. ${ }^{22}$

The height of F-actin as obtained by AFM was as expected between 6 and $8 \mathrm{~nm}$, and a cross section revealed a full width at half-maximum (FWHM) height of about $10 \mathrm{~nm}$ (Figure 2d). 

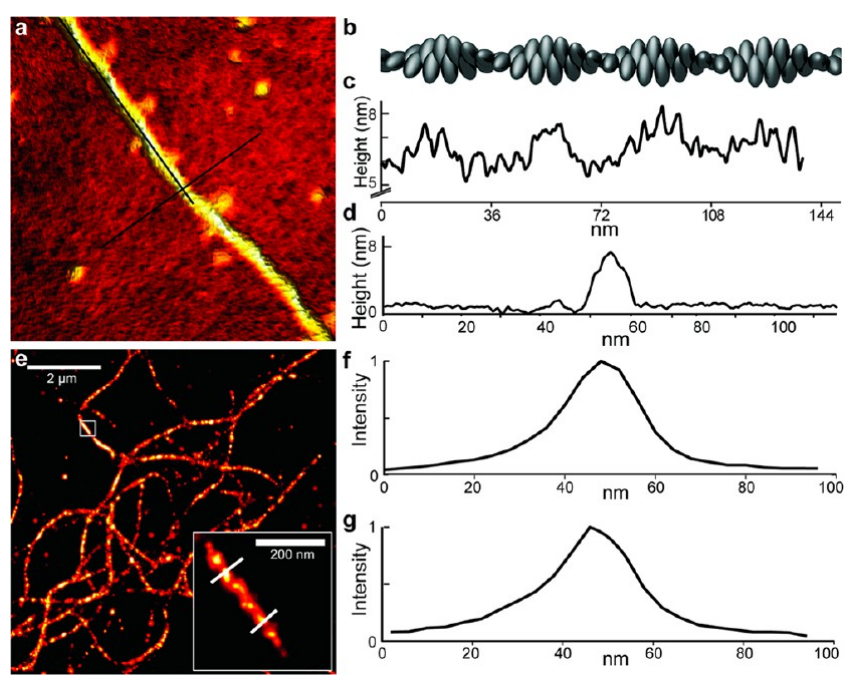

Figure 2. Independent performance of AFM and ASTORM on the combined AFM/SMLM system. (a) AFM image of F-actin deposited on an APTES coated glass coverslip $(200 \mathrm{~nm} \times 200 \mathrm{~nm})$. (b) 3D model of F-actin showing its apparent helical structure, which is observed by AFM as well. (c) Height profile of F-actin extracted from AFM data, along the filament (profile line indicated in a). The length of one helical turn is $36 \mathrm{~nm}$. (d) Profile perpendicular to the F-actin having a full width at the base of $<15 \mathrm{~nm}$. (e) Probability map of Factin labeled with phalloidin-alexa647 imaged with dSTORM, with a selected filament zoomed in (inset). Pixel size full image: $10 \mathrm{~nm}$, inset: $4 \mathrm{~nm}$. ( $\mathrm{f}$ and $\mathrm{g}$ ) Intensity profiles corresponding to the lines in the inset, normalized to the maximum intensity in the profile. The FWHM of the profiles are $\approx 24 \mathrm{~nm}$.

This result demonstrates that the performance of the AFM is not deteriorated by the fact that it was recorded on the combined SMLM/AFM system.

To assess the image quality obtained by SMLM on this combined system, we imaged F-actin labeled with phalloidinAlexa647, since the Alexa647 dye has been reported to give optimal SMLM image quality. ${ }^{26}$ Figure 2e shows a dSTORM image with a mean photon count of about 5500 (Supporting Figure 1a) and a mean localization precision of $12.5 \mathrm{~nm}$, comparable to the values reported in the literature. ${ }^{26}$ Actin filaments that have a cross-section profile with a FWHM of $\sim 24$ $\mathrm{nm}$ are visible (Figure $2 \mathrm{e}-\mathrm{g}$ ). Our measurements demonstrate that a high image quality is obtainable by both techniques individually even when performed on the combined instrument.

The procedure for correlated image acquisition is depicted in Figure 3a through h. Since Alexa647 emission deteriorates after exposure to the AFM laser (Supporting Figure 2a-c), phalloidin-Atto488 was used instead to label F-actin for the correlative experiment. This significantly reduced the bleaching of the dye, although some bleaching still occurs (compare Figure $3 \mathrm{~b}$ and $\mathrm{e}$ ), but also reduced the photon count and therefore the ultimately achievable localization precision. We recorded the AFM image before recording the dSTORM image, since the dSTORM seemed to degrade the structural integrity of the actin filaments (for details see Supporting Figure 10). By minimizing the AFM peak forces to as low as 50 $\mathrm{pN}$, the sample remained intact for the subsequent dSTORM image acquisition, which is a prerequisite for recording correlative images. After the AFM image was recorded, the buffer was exchanged, and phalloidin-atto488 was forced into a triplet state by means of the chemical reducing agent $\mathrm{NaBH}_{4}$ (Figure 3f). Subsequently, the reducing agent was washed and the buffer exchanged with the dSTORM imaging buffer containing an oxygen scavenging system to ensure optimal blinking behavior. Then, the dSTORM image was acquired (Figure $3 \mathrm{~h}$ ). Figure $3 \mathrm{i}$ through $\mathrm{k}$ show the resulting AFM images and the dSTORM image, with the squares in Figure 3k indicating the areas where the AFM images $i$ and $j$ were taken. Similar results are displayed in Supporting Figure 3.

The effects of various forms of aberrations, primarily spherical aberrations in SMLM, ${ }^{13}$ are minimized by performing accurate transformation and overlay (detailed in the Supporting Information and Supporting Figure 4). The correlative images allow comparison as well as provide complementary information about the sample. Comparing the width of the actin bundles as measured by AFM and by dSTORM, we find that dSTORM provides comparable values to AFM. On the ability to distinguish two diverging bundles of filaments, AFM provides clearer information than dSTORM (Figure $4 a-c$ ). The height and width of the actin filament bundles measured by AFM was 14 and $65 \mathrm{~nm}$ FWHM, respectively. The FWHM of dSTORM was $94 \mathrm{~nm}$ and of TIRF $271 \mathrm{~nm}$ for the filament bundles.

In addition to direct comparison, the two techniques provide complementary information about the sample. The dSTORM image reveals a nonhomogeneous intensity along the filament which is often attributed to local differences in labeling along the filament. The correlated AFM measurements however suggest that the fluctuation in localization densities is partially due to the presence of actin bundles rather than single filaments. The tendency of F-actin filaments to bundle up ${ }^{27}$ therefore likely contributes to the local differences of localizations recorded by dSTORM along the filament (Figure $4 \mathrm{~h}$ ). We indeed observed a correlation between the height of the F-actin and the number of localizations recorded at that particular location (Figure 4i). Along a single filament with a height of around $8 \mathrm{~nm}$ about 8 localizations are detected per line scanned by AFM (corresponding to $\sim 12 \mathrm{~nm}$ in length). On the other hand, in areas where an increased height was measured $(\sim 12-14 \mathrm{~nm})$, the number of dSTORM localizations is 1.5 times higher than observed on a single filament.

After correlation protocols have been established, we used the combination of the AFM and SMLM to extract complementary information about two different aspects of one and the same biological sample. For this we extended our method to samples including chemically fixed bacterial cells (Figure $4 j$ ) and mammalian cells (Supporting Figure 6). Escherichia coli (E. coli) expressing the fusion protein RNPmEOS2 was measured by AFM and subsequently by PALM. We chose PALM over dSTORM for these experiments since PALM can be performed with lower laser power, which is better suited for eventually extending the technique to live cell imaging. The AFM provides the 3D morphology of the bacteria, while PALM provides the expression level and the $2 \mathrm{D}$ projection of the spatial distribution of the fusion protein. Interestingly, one of the bacteria showed no presence of the fusion protein in a part of the PALM image. The AFM image clearly shows that this bacterium is situated slightly on top of two other bacteria. We hypothesize therefore that the fusion proteins of this particular bacteria were not mapped because the bacterium was out of the illumination volume covered in TIRF conditions. This is a good example where the combination of the two techniques yields a clearer interpretation of the microscopy data than PALM alone. However, the conventional PALM we use does not provide $3 \mathrm{D}$ information on the location 

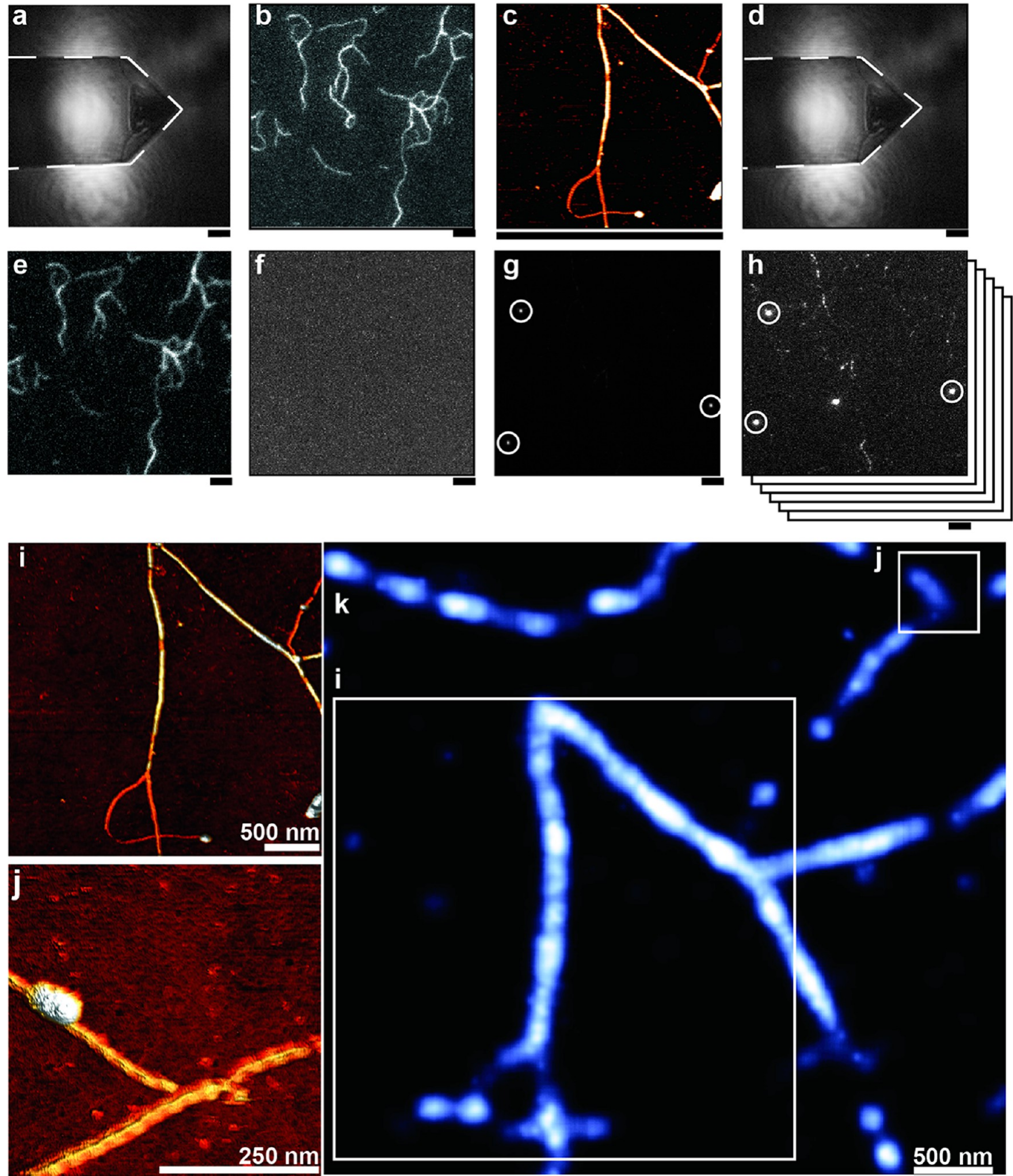

$500 \mathrm{~nm}$

Figure 3. Experimental procedure and representative correlated AFM/dSTORM images. Experimental procedure (a-h): (a) The AFM cantilever is centered in the optical field of view of the camera by translating the inverted microscope in $x$ - and $y$-direction. (b) The sample is moved, and a region of interest is selected. (c) The AFM image is acquired. (d) To determine the center of the scan another optical image is taken to account for an applied offset during AFM image acquisition. (e) Then, another TIRF image of the sample was taken and the illumination condition was optimized. (f) The fluorescent labels (phalloidin-Atto488) are quenched chemically and forced into a dark state by exchanging the buffer containing $10 \mathrm{mM}$ $\mathrm{NaBH}_{4}$. (g) The buffer of the sample is exchanged with optimal dSTORM buffer, and beads are added to be used as fiducial markers during the image reconstruction. (h) dSTORM images are acquired. Scale bars below images $(\mathrm{a}-\mathrm{h}$ ) are $3 \mu \mathrm{m}$. (i and j) AFM images, (k) reconstructed dSTORM image in probability map representation. ( $i$ and $j$ ) Areas correlated with AFM. 

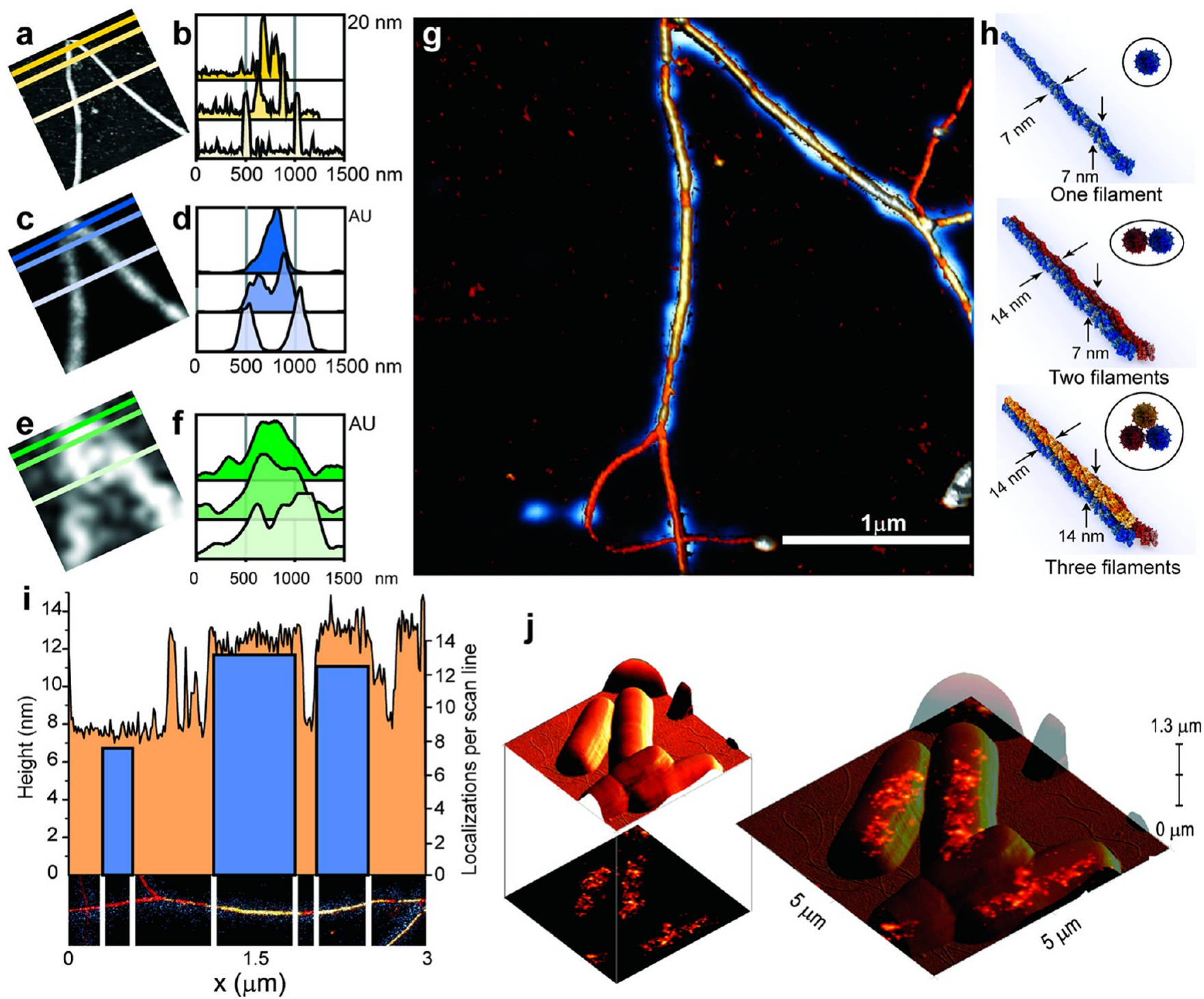

Figure 4. Comparison of AFM, dSTORM, and TIRF imaging resolution from correlative imaging. (a, c, and e) AFM, dSTORM, and TIRF images, respectively $(1.5 \mu \mathrm{m} \times 1.5 \mu \mathrm{m})$, of correlated filaments. Lines and colors indicate the location of profiles shown in (b, d, and f). (g) Overlay of dSTORM probability map (blue) and 3D rendered AFM image (yellow-brown). (h) Different F-actin arrangements suggested based on the AFM data. (i) Orange: Maximum height projection of the AFM section shown below the plot. Blue: Number of localizations detected in regions between the white lines per line scan of the AFM. In higher areas more localizations are observed. (j) Correlative AFM/PALM image of E. coli bacteria expressing RNA polymerase-mEos2.

of the fluorescence signals with respect to the morphology of the cell. Recent advancements in $3 \mathrm{D} \mathrm{PALM}^{28}$ would further leverage the power of the correlated technique.

The same technique can be applied to correlated AFM/ PALM measurements on mammalian cells. Supporting Figure 6 shows a fixed mouse embryonic fibroblast expressing the fusion protein paxillin-mEos 2 , which is part of the focal adhesion complexes that form contact with the substrate.

Performing correlated SMLM and AFM measurements within biologically relevant media not only allows for direct comparison of the two techniques but also allows us to learn more about each individual technique and to augment the weaknesses of one with the strength of the other. In the case of the actin filaments, AFM provided a reference for the location and height of the filaments that could be directly compared to the labeling density fluctuations along the filaments as observed by SMLM. This showed that the labeling fluctuations originate partially from the presence of double and triple filaments
(Figure $4 \mathrm{~h}$ and i) and is therefore not solely due to insufficient labeling as it was incorrectly assumed. On the other hand, in areas along the filament revealed by AFM to be a single filament, the fluctuations can now be unequivocally attributed to insufficient binding of tagged phalloidin. This dual information makes the comparison between different labeling protocols much more effective and accurate. On the other hand, the fact that the number of localizations per scan-line correlates well with the AFM height (and therefore the number of filaments) suggests that the localization intensity could eventually be used for quantifying the number of actin filaments in a bundle in situations where no AFM information can be obtained such as in whole cell imaging.

Thus, far, we have chosen our sample so that a direct comparison of the two techniques is possible and we also showed the potential of revealing complementary information in fixed bacteria and mammalian cells. Now that the correlation between the two techniques has been established, they can be 

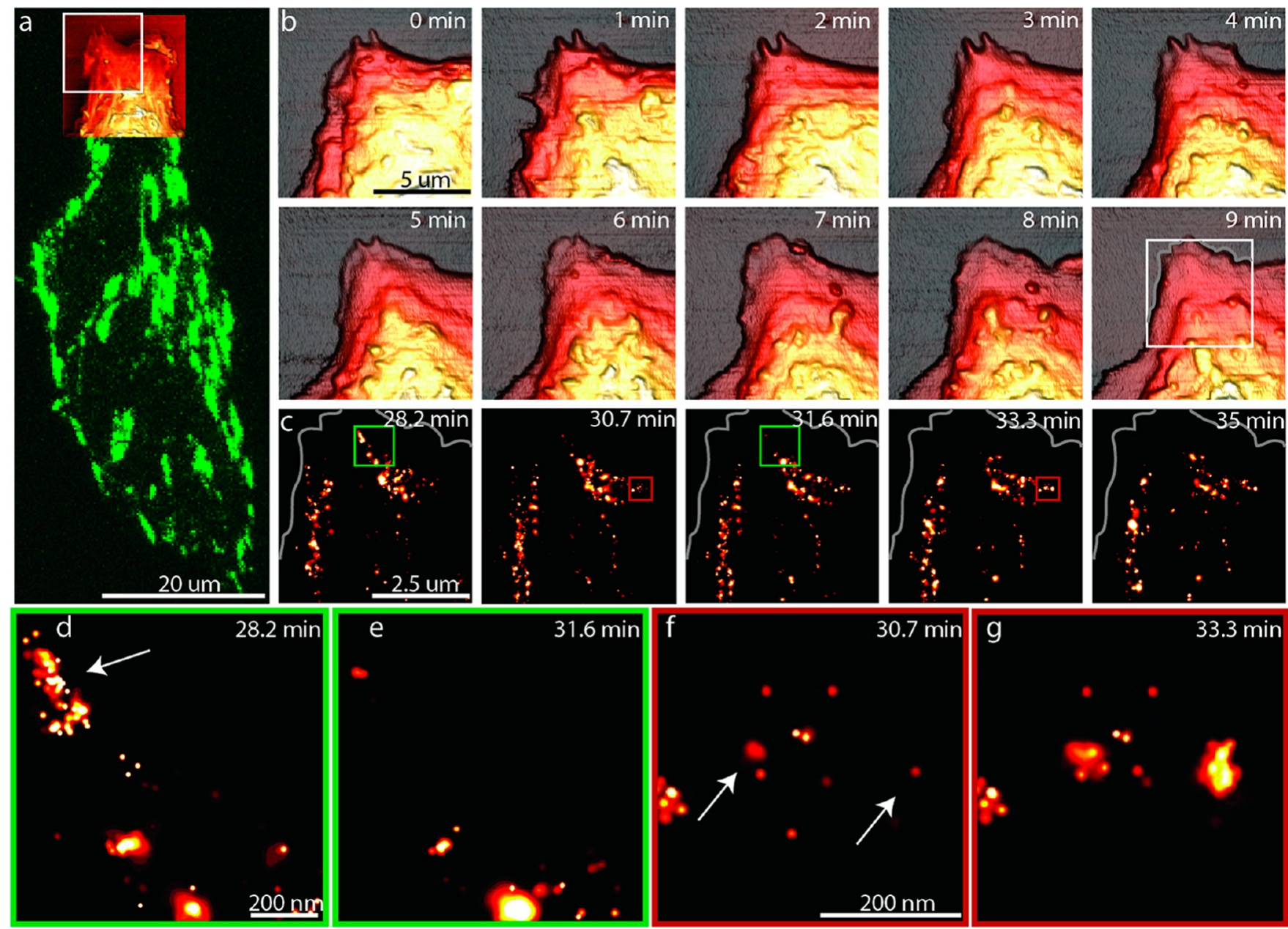

Figure 5. Live-cell time-resolved AFM/PALM on mammalian cell. (a) CHO-K1 cell transiently expressing a paxillin-mEos2 construct imaged in its off-state under TIRF illumination. Overlaid is an AFM overview image from the upper part of the cell. The white square outlines the area zoomed in for the AFM time-series shown in b. (b) Time-resolved AFM sequence of the leading edge of the cell, showing the normal cell behavior of filopodia protrusion with subsequent lamellopodia extension. The frame rate is $1 \mathrm{~min}^{-1}$. The white square in the AFM image recorded after 9 min marks the area of subsequent PALM images shown in c. (c) Live-cell PALM time series showing the reorganization of the paxillin-mEos2 clusters. (d) Zoom-in of the green square outlined in c at $28.2 \mathrm{~min}$. (e) The same area as in d at $31.6 \mathrm{~min}$. (f) Zoom of the area outlined in red in (c) in the image taken at $30.7 \mathrm{~min} .(\mathrm{g})$ Zoom of the same area as in part $\mathrm{f}$ at $33.3 \mathrm{~min}$. While the AFM images show the dynamics of the cell membrane, the PALM series monitors the changes in the paxillin-mEos 2 clusters. In $\mathrm{d}$ and e, paxillin clusters disappear from the top left corner (arrow). New paxillin clusters are formed within $3 \mathrm{~min}$ at the site shown in $\mathrm{f}$ and $\mathrm{g}$ (arrows).

reliably used to complement each other in applications where the protein specific SMLM information has to be put in a 3D cellular or mechanical context. This will be especially important for the recent and ongoing developments in 3D SMLM. ${ }^{29-32}$ Since we perform our AFM experiments in physiological solution, it is even possible to perform live cell imaging and nanomechanical stimulation using the AFM cantilever. Using the combination of live cell $\mathrm{AFM}^{33-35}$ and live cell SMLM, ${ }^{36}$ one could observe the reaction of the cells to nanomechanical stimuli with unprecedented resolution. A major concern for live cell imaging is the image acquisition speed of both SMLM and $\mathrm{AFM}$, as they both traditionally require tens of minutes to obtain a high quality image. With the development of high speed AFM by Ando et al. ${ }^{4}$ and the pioneering work in highspeed SMLM by Zhuang et al., ${ }^{36,37}$ real time nanoscale imaging of living cells is within reach. Our combined AFM/SMLM system is already capable of such experiments since live cell PALM has already been reported, ${ }^{11}$ and the AFM we used is capable of imaging live cells within a minute or less. ${ }^{38}$ Figure 5 shows such time-resolved AFM and PALM image sequences of live $\mathrm{CHO}-\mathrm{K} 1$ cells in physiological environment. From the whole cell shown in Figure 5a, we chose the leading edge (upper corner) for time-resolved analysis (Figure $5 b$ and $c$ ). AFM images (Figure $5 \mathrm{~b}$ ) were recorded at $1 \mathrm{~min}$ intervals showing filopodia already extended with subsequent following of the lamellipodium. PALM images shown in Figure 5c were recorded directly after AFM imaging, and the cell edge from the last image in the AFM sequence is shown for reference in the PALM images. Fluorescent frames were recorded at a rate of 20 frames/s and processed 5000 frames at a time with a "running average" of 1000 frames to create each PALM image (see methods, Supporting Figure 11 and Supporting movie 1 for details). Figure $5 \mathrm{~d}-\mathrm{g}$ show zoom-ins of regions depicting paxillin clusters in focal adhesions. In $\mathrm{d}$ and e, a paxillin cluster disappears at one place, while in $\mathrm{f}$ and $\mathrm{g}$ new ones appear.

The dynamic changes we observed in both the AFM and PALM images and the complementary information they provide demonstrate the potential of correlated AFM/PALM imaging of live cells. It should be noted that in these experiments the AFM and PALM data were recorded in a 
correlated fashion, but not simultaneously. A better integration of the AFM operating software with the PALM acquisition software would allow for truly simultaneous imaging. While many of the experimental conditions for routine experiments still have to be worked out, we expect this to become a truly enabling method in the study of mechanobiology and mechanotransduction. ${ }^{39,40}$

Materials/Methods. Choice of Labeling Strategy. What fluorescent labeling strategy is best suited depends on the kind of correlated experiment that is performed. In the case of the actin filaments we imaged the same aspect of a sample with both modalities. In that case it is imperative that the fluorescent labeling does not interfere with the sample structure as measured by AFM. In such a case it is preferential to use a small intercalating dye such as fluorescently labeled phalloidin. When one images different aspects of a sample with the two techniques (such as we did in the combined imaging of cells), a broader choice of labeling strategies is available such as antibody labeling or genetic expression of fluorescent fusion proteins. We chose the latter for live cell imaging as it allows for labeling of intracellular structures without permeabilizing the cell membrane. In all cases, it is essential however that the AFM laser does not bleach the fluorophores.

F-Actin. F-actin has been polymerized from G-actin following the manufacturer's protocol (BK003, Cytoskeleton, Inc.) and stored on ice subsequently. To $5 \mu \mathrm{L}$ of a G-actin aliquot $(200 \mu \mathrm{M}) 45 \mu \mathrm{L}$ prechilled general actin buffer was added, ending up with $20 \mu \mathrm{M}$ G-actin in $5 \mathrm{mM}$ Tris- $\mathrm{HCl} \mathrm{pH}$ 8.0, $50 \mathrm{mM} \mathrm{KCl}, 0.2 \mathrm{mM} \mathrm{CaCl} \mathrm{Cl}_{2}$. Next, $5 \mu \mathrm{L}$ of $10 \times$ polymerization buffer was added, resulting in a final concentration of $50 \mathrm{mM} \mathrm{KCl}, 2 \mathrm{mM} \mathrm{MgCl}, 1 \mathrm{mM} \mathrm{ATP}$, and incubated at room temperature to polymerize for $2 \mathrm{~h}$ before storing on ice. Actin was stored up to one month.

E. coli. E. coli bacteria expressing RNA polymerase labeled with $\mathrm{mEos} 2^{41}$ were kindly provided by Dr. Mike Heilemann. The bacteria were grown in lysogeny broth (LB) medium supplemented with ampicillin $(0.1 \mathrm{mg} / \mathrm{mL})$ at $37{ }^{\circ} \mathrm{C}$ in an orbital shaker overnight. Then, $100 \mu \mathrm{L}$ of bacteria solution was added to $4 \mathrm{~mL}$ of fresh medium and incubated again for $3 \mathrm{~h}$. Subsequently, $1 \mathrm{~mL}$ was pelleted by centrifugation, and the supernatant was discarded. The pellet was resuspended in deionized water, and the washing step was repeated once more. The pellet was eventually resuspended in $400 \mu \mathrm{L}$ of deionized water and used for subsequent sample preparation on the prepared glass coverslip.

Glass Coverslip. $25 \mathrm{~mm}$ diameter round glass coverslips (72225-01, Electron Microscopy Sciences) were cleaned by piranha etch $\left(\mathrm{H}_{2} \mathrm{SO}_{4}\right.$ and $\mathrm{H}_{2} \mathrm{O}_{2}$ at a ratio of 2:1) on a heating plate for at least $20 \mathrm{~min}$ and then left to cool down. Then, the coverslips were thoroughly rinsed with water and blown dry by a nitrogen stream. On coverslips prepared for the F-actin sample, $500 \mu \mathrm{L}$ of water (Milli-Q) containing $0.5 \%(\mathrm{v} / \mathrm{v}$ ) APTES (440140, Sigma-Aldrich, Switzerland) was deposited and incubated for $10 \mathrm{~min}$ before rinsing with water. Coverslips were then dried at $65^{\circ} \mathrm{C}$ for $1 \mathrm{~h}$ in vertical position. Coverslips were prepared freshly on the day of the experiment.

Cleaned coverslips used to deposit E. coli were immersed for $10 \mathrm{~min}$ in a solution containing $0.05 \mathrm{mg} / \mathrm{mL}$ poly-L-lysine (PLL) hydrobromide (Sigma, P1524) and $10 \mathrm{mM}$ Tris at $\mathrm{pH} 8$. Coverslips were then dried in a vertical position at room temperature overnight and used within a week.

Cleaned and coated coverslips were glued into a custommade holder before the respective sample was deposited.
Sample Deposition and Imaging Buffers. F-actin samples were prepared as follows: $3 \mu \mathrm{L}$ of phalloidin-Alexa647 (A22287, Life Technologies) or phalloidin-Atto488 (49409, Sigma-Aldrich, Switzerland) and $1 \mu \mathrm{L}$ of polymerized F-actin was added to $45 \mu \mathrm{L}$ of Buffer A ( $2 \mathrm{mM} \mathrm{MgCl}_{2}, 1 \mathrm{mM}$ EGTA, $20 \mathrm{mM}$ imidazole- $\mathrm{HCl}, \mathrm{pH} 7.6)$ and incubated in darkness for $10 \mathrm{~min}$. Then $100 \mu \mathrm{L}$ Buffer A was added, and $50 \mu \mathrm{L}$ of this was deposited on a APTES-functionalized coverslip. Another $150 \mu \mathrm{L}$ Buffer A was added to the sample and incubated for 5 min. Additionally, up to $1 \mathrm{~mL}$ of Buffer A was added for AFM experiments.

E. coli samples were prepared as follows. A drop of concentrated bacteria solution was deposited onto a PLLcoated coverslip, and bacteria were left to adhere for $30 \mathrm{~min}$ before the coverslip was gently washed with water. After imaging by AFM in water, the sample was washed with PHEM buffer (60 mM Pipes, $25 \mathrm{mM}$ HEPES, $10 \mathrm{mM}$ EGTA, and 2 $\mathrm{mM} \mathrm{MgCl} 2$ at $\mathrm{pH}$ 6.9) and fixed with $4 \%$ paraformaldehyde in PHEM buffer for $30 \mathrm{~min}$ without changing the position of the sample. Subsequently, the sample was washed with PHEM buffer 3 times, and PALM images were acquired.

$\mathrm{CHO}-\mathrm{K} 1$ Cells. CHO-K1 cells were grown in an incubator at $37{ }^{\circ} \mathrm{C}, 5 \% \mathrm{CO}_{2}$ in DMEM-F12 supplemented with $10 \%$ (v/v) FBS and $1 \%$ antibiotics to $70 \%$ confluency prior to transfection. $4 \times 10^{5}$ cells were transfected with $2 \mu \mathrm{g}$ of plasmid DNA containing the paxillin-mEos 2 sequence with a Neon electroporation system. Cells were then seeded in a 6-well plate with DMEM supplemented with FBS only. The following day they were transferred onto plasma cleaned fibronectin coated $25 \mathrm{~mm}$ diameter glass coverslips and grown for another 2-24 h. Prior to the experiment the cells on the coverslip were transferred on a home-built coverslip heater shown in Supporting Figure 9 to keep them at $37^{\circ} \mathrm{C}$ and the medium was exchanged to DMEM without phenol red buffered with $25 \mathrm{mM}$ Hepes. As imaging took less than $35 \mathrm{~min}$, it was not necessary to have a controlled $\mathrm{CO}_{2}$ environment. For longer term imaging we suggest putting a $\mathrm{CO}_{2}$ cage around the sample including the AFM, or use the commercially available perfusion chamber for the FastScan Bio AFM.

AFM. AFM experiments were conducted with a Dimension FastScan (Bruker, USA) mounted on a custom-built support structure on top of an inverted optical microscope (IX71, Olympus, Japan). BL-AC40TS-C2 cantilevers (Olympus, Japan) with a nominal spring constant of $0.09 \mathrm{~N} / \mathrm{m}$ and a resonance frequency of $110 \mathrm{kHz}$ were used to image the F-actin samples. Images were taken in PeakForce QNM mode at a maximal force $<100 \mathrm{pN}$, at a resolution of $512 \times 256$ pixels. $E$. coli samples were imaged using Scanasyst-Fluid cantilevers (Bruker) with a nominal spring constant of $0.7 \mathrm{~N} / \mathrm{m}$ and a resonance frequency of $150 \mathrm{kHz}$. For the live-cell experiment on CHO-K1 cells Fastscan-D cantilevers (Bruker) with a nominal spring constant of $0.25 \mathrm{~N} / \mathrm{m}$ and a typical resonance frequency in water of $110 \mathrm{kHz}$ were used. Images were taken in tapping mode at a resolution of $512 \times 128$ and a frame rate of 1 $\mathrm{min}^{-1}$. AFM data was processed with standard SPM software. Supporting Figure 7 displays 2D AFM height images of the 3D representations shown in the main figures. The reconstruction of the tip used in Figure 3 as obtained by the software Nanoscope Analysis (Bruker, USA) is shown in Supporting Figure 8. More details are provided in the text of the Supporting Information.

dSTORM. For the imaging with Alexa-647, the sample was imaged in the buffer conditions described in Olivier et al. ${ }^{42} 2$ 
$\mathrm{mL}$ of buffer ( $\mathrm{pH} 7.5$ ) was composed of: $1.685 \mathrm{~mL}$ of PBS, 200 $\mu \mathrm{L}$ of Tris (100 mM pH 7.4), $20 \mu \mathrm{L}$ of $1 \mathrm{M}$ mercaptoethylamine stock $\mathrm{pH} 8$ (MEA, Sigma; $\mathrm{pH}$ adjusted with glacial acetic acid $100 \%$ ), $7 \mu \mathrm{L}$ of $\beta$-mercaptoethanol (M6250, Sigma), $20 \mu \mathrm{L}$ of $200 \mathrm{mM}$ cyclooctatetraene (COT) stock made from $1.27 \mathrm{~mL}$ of DMSO and $30 \mu \mathrm{L}$ of COT (Sigma), $50 \mu \mathrm{L}$ of $100 \mathrm{mM}$ protocatechuic acid (PCA, Sigma) at $\mathrm{pH} 9$ (adjusted with $\mathrm{KOH}$ ), and $20 \mu \mathrm{L}$ of $5 \mu \mathrm{M}$ protocatechuic dioxygenase (PCD, Sigma) stock stored in $50 \%$ glycerol in $50 \mathrm{mM} \mathrm{KCl}, 1 \mathrm{mM}$ EDTA, and $100 \mathrm{mM}$ Tris- $\mathrm{HCl} \mathrm{pH} 8$.

For the correlative imaging with ATTO-488, the following protocol was used. After AFM imaging, the sample was washed $3 \times$ with PBS, leaving $300 \mu \mathrm{L}$ PBS. $600 \mu \mathrm{L}$ of PBS containing $0.5 \mathrm{mg} / \mathrm{mL} \mathrm{NaBH}_{4}$ was added to a final concentration of 10 $\mathrm{mM} \mathrm{NaBH} 4$ and incubated for at least $5 \mathrm{~min}$ or until the fluorescence was quenched. Then the sample was washed with Tris/Trolox buffer $\mathrm{pH} 8$ (100 mM Tris, $1 \mathrm{mM}$ Trolox), leaving $250 \mu \mathrm{L}$ of buffer on the sample. Next, $750 \mu \mathrm{L}$ of buffer containing glucose, catalase, and glucose oxidase was added, resulting in a final concentration of $0.5 \mathrm{mg} / \mathrm{mL}$ glucose oxidase, $40 \mu \mathrm{g} / \mathrm{mL}$ catalase and $5 \%(\mathrm{w} / \mathrm{v})$ glucose. Additionally, $3 \mu \mathrm{L}$ of $100 \mathrm{~nm}$ fluorescent beads (Tetraspeck, Life technologies) was added to the sample.

The dSTORM measurements were performed with a TIRF objective (APON 60X TIRF, Olympus, after a further magnification of $2 \times$, yielding a pixel size of $133 \mathrm{~nm}$ ) and appropriate optical filters (dichroic: F73-866; emission filter: F72-866, AHF Analysetechnik AG, Germany). Illumination was provided by a monolithic laser combiner (MLC400B, Agilent, UK), and the optical fiber output of the MLC400B was coupled into an optical system (TIRFM Illuminator, Olympus, Japan) in order to generate TIRF illumination. Images were recorded with an iXon Ultra 897 (Andor) EMCCD camera. The power of the $488 \mathrm{~nm}$ laser at the fiber output was set to $80 \mathrm{~mW}$ and that of the $647 \mathrm{~nm}$ laser at a maximum of $140 \mathrm{~mW}$, the gain of the EMCCD camera was set to 200, and images were taken with an exposure time of $20 \mathrm{~ms}$.

PALM. The fixed MEF cells were imaged in PHEM buffer at room temperature. The live $\mathrm{CHO}-\mathrm{K} 1$ cells were imaged in 25 $\mathrm{mM}$ Hepes buffered DMEM at $37{ }^{\circ} \mathrm{C}$. More details can be found in the Supporting Information.

The PALM measurements were performed with the same setup as described in the dSTORM section. The emission light coming from mEos 2 was separated from the illumination light using a combination of a dichroic mirror (493/574 nm BrightLine, Semrock) and an emission filter (405/488/568 nm StopLine, Semrock). An optical system (DV2, Photometrics) equipped with a dichroic mirror (617/73 nm BrightLine, Semrock) was placed between the EMCCD camera and the microscope frame. The DV2 splits the emission light from the on- and off-state of the mEos 2 and sends each color to a separate half of the EMCCD camera chip.

In order to find a cell in the sample, the mEos 2 in the offstate was visualized in the green channel by excitation of 488 $\mathrm{nm}$ with $5 \mathrm{~mW}$ power at the MLC400B output. Before imaging of the mEos 2 in the on-state, fiduciary beads (100 nm gold nanospheres, Corpuscular) were added to the sample. The gold beads that did not sediment after $5 \mathrm{~min}$ are removed by washing $1 \times$ with PHEM for the MEF cell experiment. Imaging of the mEos 2 in the on-state was performed in the red channel by excitation of $561 \mathrm{~nm}$ with $30 \mathrm{~mW}$ power at the MLC400B output. During imaging, the mEos 2 in the off-state was activated by $405 \mathrm{~nm}$ with a gradually increasing power at the
MLC400B output, ranging for $0 \mathrm{~mW}$ to $2 \mathrm{~mW}$. The gain of the EMCCD camera was 200, and the exposure time was $50 \mathrm{~ms}$.

Reconstruction. The estimation of the single molecule positions from the images and the rendering as a probability map were done by the code (MATLAB, MathWorks) kindly provided by Dr. Eric Betzig, using the same algorithm described in Betzig et al. ${ }^{6}$ The localization precision was estimated by means of the expression provided in Mortensen et al., ${ }^{43}$ also incorporating the precision loss due to the use of the EMCCD camera. The factor to convert $\mathrm{ADC}$ values to photon counts was calibrated as explained elsewhere. ${ }^{44}$ The drift during imaging was corrected using either fiducial marker traces or using the correlation between frames. ${ }^{45}$ The dSTORM image displayed in Figure 2 was obtained from 16000 frames of imaging, and the one in Figures 3 and 4 from 60000 frames. For Figure 2, only localizations with a precision between 2 and 30 $\mathrm{nm}$, and a photon count between 300 and 50000 were considered. For Figures 3 and 4, a localization precision cutoff of $50 \mathrm{~nm}$ and a minimum photon count of 100 were used. The PALM image displayed in Figure 4j was obtained from 62000 frames of imaging and that in Supporting Figure 6 from 27000 frames. Localizations in both images were plotted if the corresponding mEos 2 image contained at least 100 photons and if the localization precision was between 3 and $30 \mathrm{~nm}$. The PALM time sequence (Figure 5c and Supporting Movie 1) was obtained from 14000 frames of imaging, with each individual PALM image in the sequence obtained from a subset of 5000 frames. The time sequence (Supporting Movie 1) was obtained by shifting 1000 (Supporting Movie 1) frames of imaging between each PALM image (for details see Supporting Figure 11). In Figure 5c selected frames of the sequence are shown. Only mEos 2 localizations with a precision between 2 and 40 $\mathrm{nm}$ and a photon count of at least 100 were plotted.

Overlay. Overlay between the AFM image and the STORM localizations was done by estimating an affine transformation matrix between the two by minimizing a cost function based on the overlap between the two images. More details are provided in the text of the Supporting Information.

\section{ASSOCIATED CONTENT}

\section{S Supporting Information}

Supporting Information include additional correlative AFM/ dSTORM and AFM/PALM images. Detailed explanation of the overlay procedure. $2 \mathrm{D}$ representations of the AFM images shown in the figures. Further characterizations of the dSTORM dyes, their usability for correlative SMLM/AFM approach, as well as the schematic of the heating holder used for the live-cell experiment. The Supporting Information is available free of charge on the ACS Publications website at DOI: 10.1021/ acs.nanolett.5b00572.

\section{AUTHOR INFORMATION}

\section{Corresponding Authors}

*E-mail: aleksandra.radenovic@epfl.ch.

*E-mail: georg.fantner@epfl.ch.

\section{Author Contributions}

A.S. and H.D. contributed equally to the study. A.R. and G.E.F. conceived the project. P.D.O. with the help of G.E.F. and A.P.N. built the instrument. L.F prepared the in vivo specimens. A.P.N. designed and built the coverslip heating holder. R.J. and P.D.O. developed the protocol for actin filament sample preparation. A.S optimized the STORM imaging protocol and 
implemented and performed the dSTORM/AFM overlay. P.D.O and A.S. performed the dSTORM/AFM imaging experiments and analyzed the data. P.D.O and H.D performed the PALM/AFM imaging experiments and analyzed the data. P.D.O., A.R., and G.E.F. wrote the manuscript with inputs from A.S and H.D.

\section{Notes}

The authors declare no competing financial interest.

\section{ACKNOWLEDGMENTS}

This work was financially supported by FNS grants no. 200021125319, no. 20021-132206, no. 205321-134786, and no. 205320-152675 as well as the European Union's Seventh Framework Program FP7/2007-2011 under grant agreement 286146 and Eurostars E!8213 and FP7/2007-2013/ERC grant agreement 307338. A. Shivanandan was funded by a $\mathrm{PhD}$ fellowship grant from NCCBI. We thank Dr. P. Annibale for initial help with the instrument. We thank Lina Carlini for discussion, Prof. Mike Heilemann on the generous gift of bacterial strain expressing mEos2-RNAPm and Prof. Nicolas Mermod for providing CHO-K1 cells. G.E.F. thanks Dr. Chanmin Su and Bruker Nano Surfaces for technical support.

\section{REFERENCES}

(1) Henderson, E.; Haydon, P. G.; Sakaguchi, D. S. Science 1992, 257 (5078), 1944.

(2) Dufrene, Y. F.; Boonaert, C. J. P.; Gerin, P. A.; Asther, M.; Rouxhet, P. G. J. Bacteriol. 1999, 181 (17), 5350.

(3) Rotsch, C.; Braet, F.; Wisse, E.; Radmacher, M. Cell Biol. Int. 1997, 21 (11), 685-696.

(4) Kodera, N.; Yamamoto, D.; Ishikawa, R.; Ando, T. Nature 2010, 468 (7320), 72-76.

(5) Ando, T. Nanotechnology 2012, 23 (6), 062001.

(6) Betzig, E.; Patterson, G. H.; Sougrat, R.; Lindwasser, O. W.; Olenych, S.; Bonifacino, J. S.; Davidson, M. W.; Lippincott-Schwartz, J.; Hess, H. F. Science 2006, 313 (5793), 1642-1645.

(7) Hess, S. T.; Girirajan, T. P.; Mason, M. D. Biophys. J. 2006, 91 (11), 4258-72.

(8) Rust, M. J.; Bates, M.; Zhuang, X. Nat. Methods 2006, 3 (10), $793-5$.

(9) Xu, K.; Zhong, G. S.; Zhuang, X. W. Science 2013, 339 (6118), $452-456$.

(10) Szymborska, A.; de Marco, A.; Daigle, N.; Cordes, V. C.; Briggs, J. A. G.; Ellenberg, J. Science 2013, 341 (6146), 655-658.

(11) Shroff, H.; Galbraith, C. G.; Galbraith, J. A.; Betzig, E. Nat. Methods 2008, 5 (5), 417-423.

(12) Annibale, P.; Vanni, S.; Scarselli, M.; Rothlisberger, U.; Radenovic, A. PLoS One 2011, 6 (7), e22678.

(13) Annibale, P.; Scarselli, M.; Greco, M.; Radenovic, A. Optical Nanoscopy 2012, 1 (1), 9.

(14) Durisic, N.; Laparra-Cuervo, L.; Sandoval-Alvarez, A.; Borbely, J. S.; Lakadamyali, M. Nat. Methods 2014, 11 (2), 156-162.

(15) Nieuwenhuizen, R. P.; Lidke, K. A.; Bates, M.; Puig, D. L.; Grunwald, D.; Stallinga, S.; Rieger, B. Nat. Methods 2013, 10 (6), $557-62$.

(16) Chang, Y. W.; Chen, S. Y.; Tocheva, E. I.; Treuner-Lange, A.; Loebach, S.; Sogaard-Andersen, L.; Jensen, G. J. Nat. Methods 2014, 11 (7), 737-739.

(17) Kopek, B. G.; Shtengel, G.; Grimm, J. B.; Clayton, D. A.; Hess,

H. F. PLoS One 2013, 8 (10), e 77209.

(18) Monserrate, A.; Casado, S.; Flors, C. ChemPhysChem 2014, 15 (4), 647-50.

(19) Muller, D. J.; Schabert, F. A.; Buldt, G.; Engel, A. Biophys. J. 1995, 68 (5), 1681-6.

(20) Chacko, J. V.; Zanacchi, F. C.; Diaspro, A. Cytoskeleton 2013, 70

(11), 729-40.
(21) Heilemann, M.; van de Linde, S.; Schuttpelz, M.; Kasper, R.; Seefeldt, B.; Mukherjee, A.; Tinnefeld, P.; Sauer, M. Angew. Chem., Int. Ed. 2008, 47 (33), 6172-6176.

(22) Schmitz, S.; Schaap, I. A.; Kleinjung, J.; Harder, S.; Grainger, M.; Calder, L.; Rosenthal, P. B.; Holder, A. A.; Veigel, C. J. Biol. Chem. 2010, 285 (47), 36577-85.

(23) Metcalf, D. J.; Edwards, R.; Kumarswami, N.; Knight, A. E. J. Visualized Exp. 2013, DOI: 10.3791/50579.

(24) Xu, K.; Babcock, H. P.; Zhuang, X. W. Nat. Methods 2012, 9 (2), $185-188$.

(25) Dominguez, R.; Holmes, K. C. Annu. Rev. Biophys. 2011, 40 (1), 169-186.

(26) Dempsey, G. T.; Vaughan, J. C.; Chen, K. H.; Bates, M.; Zhuang, X. W. Nat. Methods 2011, 8 (12), 1027-1036.

(27) Ikawa, T.; Hoshino, F.; Watanabe, O.; Li, Y. L.; Pincus, P.; Safinya, C. R. Phys. Rev. Lett. 2007, 98 (1), 018101.

(28) York, A. G.; Ghitani, A.; Vaziri, A.; Davidson, M. W.; Shroff, H. Nat. Methods 2011, 8 (4), 327-333.

(29) Huang, B.; Wang, W. Q.; Bates, M.; Zhuang, X. W. Science 2008, 319 (5864), 810-813.

(30) Pavani, S. R. P.; Thompson, M. A.; Biteen, J. S.; Lord, S. J.; Liu, N.; Twieg, R. J.; Piestun, R.; Moerner, W. E. Proc. Natl. Acad. Sci. U. S. A. 2009, 106 (9), 2995-2999.

(31) Shtengel, G.; Galbraith, J. A.; Galbraith, C. G.; LippincottSchwartz, J.; Gillette, J. M.; Manley, S.; Sougrat, R.; Waterman, C. M.; Kanchanawong, P.; Davidson, M. W.; Fetter, R. D.; Hess, H. F. Proc. Natl. Acad. Sci. U. S. A. 2009, 106 (9), 3125-3130.

(32) Abrahamsson, S.; Chen, J. J.; Haji, B.; Stallinga, S.; Katsov, A. Y.; Wisniewski, J.; Mizuguchi, G.; Soule, P.; Mueller, F.; Darzacq, C. D.; Darzacq, X.; Wu, C.; Bargmann, C. I.; Agard, D. A.; Dahan, M.; Gustafsson, M. G. L. Nat. Methods 2013, 10 (1), 60-63.

(33) Rotsch, C.; Jacobson, K.; Radmacher, M. Proc. Natl. Acad. Sci. U. S. A. 1999, 96 (3), 921-926.

(34) Colom, A.; Casuso, I.; Rico, F.; Scheuring, S. Nat. Commun. 2013, 4, 4.

(35) Dufrene, Y. F.; Martinez-Martin, D.; Medalsy, I.; Alsteens, D.; Muller, D. J. Nat. Methods 2013, 10 (9), 847-854.

(36) Shim, S. H.; Xia, C. L.; Zhong, G. S.; Babcock, H. P.; Vaughan, J. C.; Huang, B.; Wang, X.; Xu, C.; Bi, G. Q.; Zhuang, X. W. Proc. Natl. Acad. Sci. U. S. A. 2012, 109 (35), 13978-13983.

(37) Jones, S. A.; Shim, S.-H.; He, J.; Zhuang, X. Nat. Methods 2011, 8 (6), 499-505.

(38) Slade, A.; Minne, S. C. Microsc. Microanal. 2014, 20 (SupplementS3), 2074-2075.

(39) Ingber, D. E. FASEB J. 2006, 20 (7), 811-827.

(40) Wang, Y. X.; Shyy, J. Y. J.; Chien, S. Annu. Rev. Biomed. Eng. 2008, 10, 1-38.

(41) Endesfelder, U.; Finan, K.; Holden, S. J.; Cook, P. R.; Kapanidis, A. N.; Heilemann, M. Biophys. J. 2013, 105, 172.

(42) Olivier, N.; Keller, D.; Gonczy, P.; Manley, S. PLoS One 2013, 8 (7), e69004.

(43) Mortensen, K. I.; Churchman, L. S.; Spudich, J. A.; Flyvbjerg, H. Nat. Methods 2010, 7 (5), 377-U59.

(44) Janesick, J. R. Scientific charge-coupled devices. In pie Press Monograph; SPIE Publications: Bellingham, WA, 2007.

(45) Ovesny, M.; Krizek, P.; Borkovec, J.; Svindrych, Z. K.; Hagen, G. M. Bioinformatics 2014, 30 (16), 2389-2390. 